\title{
Blind Cooperation: \\ The Evolution of Redundancy via Ignorance
}

\author{
Makmiller Pedroso
}

\begin{abstract}
One curious phenomenon of several social groups is that they are 'redundant' in the sense that they contain more cooperators than strictly needed to complete certain group tasks, such as foraging. Redundancy is puzzling because redundant groups are particularly susceptible to invasion by defectors. Yet, redundancy can be found in groups formed by a wide range of organisms, including insects and microbes. Jonathan Birch ([2012]) has recently argued that coercive behaviors might account for redundancy using insect colonies as a case study. However, microbial examples suggest redundancy can evolve without coercive behaviors. This paper formulates an explanation for redundancy that does not require targeted punishment of defectors; instead, it proposes that redundancy might be due to ignorance. Specifically, it is suggested that redundancy evolves as a by-product of selection when group members have to opt whether to cooperate or not while being blind to the strategies of others. Accordingly, possessing information about the strategies of the group members might undermine rather than facilitate cooperation within groups.
\end{abstract}

Keywords: redundancy; complex systems; cooperation; microbes; policing; public goods games; division of labor. 


\section{Contents}

1 The evolution of redundancy 2

2 Explaining redundancy via coercion 4

$\begin{array}{lll}3 & \text { Explaining redundancy via ignorance } & 7\end{array}$

3.1 Cooperation behind the Darwinian veil of ignorance $\ldots \ldots .7$

3.2 Modeling group tasks with a public goods game . . . . . . . . 10

3.3 Group tasks under harsh environments . . . . . . . . . . . . . 13

\section{The evolution of redundancy}

Social interactions often take place within sizable groups. In the case of human evolution, being able to cooperate in large groups beyond the immediate family likely proved crucial for the evolution of ethical norms (Bowles and Gintis, [2011]). Insects often form colonies equipped with sophisticated systems of division of labor (Anderson et al., [2001]). Microbes have been living in dense multicellular clusters called 'biofilms' for over three billion years (Westall et al., [2001]). Further, multicellular organisms are essentially groups of trillions of cooperative cells that are nearly free of conflicts (Queller and Strassmann, [2009]). Altogether these examples indicate that social evolution and group living consistently go hand in hand within nature.

Social groups are expected to evolve via natural selection because organisms are more likely to successfully complete certain tasks when they team up (Anderson and Franks, [2001]; Krause and Ruxton, [2002]; Calcott, [2008]). ${ }^{1}$

\footnotetext{
${ }^{1}$ Group living can also negatively impact its members. For example, living as part of groups can increase competition between group members, and groups might be more visible to predators than solitary individuals. See Krause and Ruxton ([2002], ch. 3) for further
} 
Sometimes group tasks involve an intricate system of division of labor, such as brood care in eusocial insects (Hölldobler and Wilson, [2009]). In other cases, group tasks are due to multiple individuals performing the same task. Army ants can, for example, build bridges with their own bodies (Anderson et al., [2002]). In the microbial world, bacterial cells can protect themselves from antibiotics if enough cells produce a particular type of extracellular product (Stewart and Costerton, [2001]; Flemming et al., [2016]).

Group tasks are often performed by 'redundant' groups in the sense that they have more cooperators than typically necessary to complete the task. This phenomenon is well-documented in insects, which often form colonies containing more workers than strictly needed to perform colony functions, such as foraging and caring for the brood. Additionally, the level of redundancy in insect colonies is substantial, with multiple studies reporting that over half of the workers are part of the 'reserve' labor force (Charbonneau et al., [2017]). Like insects, other types of organisms also form redundant groups, including microbes (Rainey and Rainey, [2003]) and mammals (Wilkinson, [1984]). Thus, as indicated by several studies, group redundancy is ubiquitous in nature (Charbonneau and Dornhaus, [2015]; Birch, [2017],2). ${ }^{2}$

One common type of explanation for redundancy is that it is adaptive at the group-level. In particular, redundant groups are probably more resilient to environmental fluctuations than non-redundant groups (Charbonneau et al., [2017]). The reason this occurs is because redundant groups can replace lost workers due to environmental disturbances and mobilize extra workers in moments of need. Still, this type of explanation fails to explain why individuals would cooperate in groups with a surplus of cooperators. First of all, group tasks details.

${ }^{2}$ In fact, complex systems are often redundant in a broad sense, including genomes and computer networks (Charbonneau and Dornhaus, [2015]; Nowak et al., [1997]; Albert et al., [2000]). 
are likely to be successfully completed in redundant groups and, as a result, the expected benefit gained by becoming a cooperator is negligible (Birch, [2012]). Furthermore, cooperating is costly and, sometimes, even deadly for individuals. Basically, group redundancy exacerbates the free-rider problem since the incentive to free-ride seems to increase in groups with a surplus of cooperators. Yet, redundancy has been observed in a wide range of social organisms, including microbes, insects, and mammals. ${ }^{3}$ Why is that?

This paper proposes that redundancy might be due to 'ignorance'. Specifically, it suggests that redundancy might evolve as a by-product of selection when group members are behind some form of 'Darwinian veil of ignorance' in the sense that they have to decide whether they will cooperate without knowing the strategy of the other members (Section 3). ${ }^{4}$ This explanation contrasts with an account of redundancy recently advanced by Jonathan Birch ([2012]). According to him, redundancy in some social groups, especially insect colonies, might be due to coercive behaviors. The next section discusses Birch's account in more detail.

\section{Explaining redundancy via coercion}

Jonathan Birch ([2012]) recently suggested redundancy might be due to coercive behaviors. In order to motivate his account, he draws a parallel between the puzzle posed by redundancy and the 'paradox of voting'. The paradox provides as follows (Brennan, [2016]). The probability that one's vote will change the outcome of an election is minuscule. Moreover, the costs involved in taking the time to vote seems to exceed the expected benefits of voting. Yet, people vote

\footnotetext{
${ }^{3}$ When formulated in terms of multilevel selection (Okasha, [2006]), the issue here is, even though group-level selection might favor redundancy, individual-level selection appears to suppress the evolution of redundancy. Accordingly, a satisfactory explanation for the evolution of redundancy would need to show why individual-level selection does not override group-level selection.

${ }^{4}$ The expression 'Darwinian veil of ignorance' is from Skyrms ([1996]).
} 
in large numbers. Birch ([2012]) claims that the extreme redundancy in insect colonies raises a similar type of puzzle. In addition to being costly, the contribution of any individual worker to the probability of task success is negligible in redundant groups. Still, redundancy is pervasive in eusocial insect colonies.

Kin selection theory is often used to explain social behaviors in insect colonies (Bourke, [2011]). As a result, kin selection theory is expected to provide a promising framework for explaining redundancy. According to kin selection, a gene for a cooperative trait can be favored by natural selection as long as the beneficiaries of the cooperative behavior are other individuals carrying the same gene. However, the level of genetic relatedness in insect societies is insufficient to explain the evolution of altruism in the absence of coercion (Ratnieks and Wenseleers, [2008]). Moreover, Birch ([2012]) argues that indirect benefits due to shared genes are insufficient to solve the puzzle posed by extreme redundancy. His reasoning is that even if indirect benefits are added to the picture, the puzzle posed by extreme redundancy persists because it is still the case that individual workers 'incur huge direct costs in return for extremely small expected indirect benefits' (Birch, [2012], p. 375). That is, appealing to indirect benefits does not change the fact that the costs seem to outweigh the benefits of cooperating in redundant groups. Because of this, Birch contends that an additional mechanism is needed to tip the scale in favor of cooperation.

Birch ([2012]) proposes that within-group coercion might have enabled the evolution of redundancy. Birch's proposal is based on empirical studies that report that altruism in insect colonies can be enforced by worker policing (Ratnieks and Wenseleers, [2008]). According to these studies, even though workers in such colonies can defect by laying their own eggs, some workers police the colony by eating the eggs laid by other workers, but not the eggs laid by the queen. In this way, policing might tip the scale in favor of cooperation by mak- 
ing defection too costly which, in turn, enables the evolution of redundancy in insect colonies. ${ }^{5}$ In Birch's words:

When an effective coercive regime is in place, large numbers of workers may stably participate in large-scale cooperative tasks even when the expected inclusive fitness benefit conferred by their own individual contributions is extremely small, because the cost of participating is fully counterbalanced by the costs imposed on defectors. The effect of systematic coercion is thus to modify the selective environment in such a way as to make the evolution of extreme redundancy possible (Birch, [2012], p. 377).

It is unclear whether Birch's account is sufficient to explain redundancy in the microbial world. Microbial groups often rely on public goods, such as enzymes, which are costly to produce but enhance the fitness of neighboring cells. Similar to insect colonies, microbial groups can also be redundant in the sense that they can possess more public good producers (cooperators) than strictly necessary to maintain the group functions. For instance, biofilms can persist even when some of their inhabitants are free-riders and do not contribute to the biofilm construction (Rainey and Rainey, [2003]; Vlamakis et al., [2008]). Birch's explanation is compelling in the case of insect colonies largely because the insects' ability to coerce defectors is well-documented. Although microbes can punish free-riders by producing bacteriocins, such as colicins in Esherichia coli, this type of coercion is expected to exist under limited conditions in microbes (Travisano and Velicer, [2004]). ${ }^{6}$ In other words, targeted punishment is

\footnotetext{
${ }^{5}$ Although Birch ([2012]) doubts that appealing to indirect benefits is sufficient to explain the evolution of redundancy, he does claim that coercive behaviors may have evolved via kin selection.

${ }^{6}$ In order to engage in policing, the bacteriocins need to harm cheaters but not cooperators. This type of policing could evolve via linked 'greenbeard alleles' (Dawkins, [1976]) that code for the production of the toxin and the identification tag, as illustrated by the genes that code for colicin production in E. coli (Travisano and Velicer, [2004]). Nevertheless, greenbeard
} 
unlikely to be the rule in the microbial world.

Microbial groups thus suggest that redundancy can evolve without the presence of coercive behaviors in the group. Following this lead, the next section suggests that redundancy can evolve in the absence of coercion as long as the group members possess limited information about the proportion of cooperators in the group. To put it simply, the next section proposes that redundancy can be due to ignorance.

\section{Explaining redundancy via ignorance}

\subsection{Cooperation behind the Darwinian veil of ignorance}

The benefit generated by completing a group task is typically not the sum of the contribution of each cooperator in the group (Birch, [2017]; Archetti and Scheuring, [2012]; Pedroso, [2018]). In particular, when there are enough cooperators to complete a task, further increasing the number of cooperators will likely not produce as much benefit due to diminishing returns. This might happen because the benefit conferred by a resource is constrained by the individual's ability to utilize the resource. For example, the higher the amount of blood stored by a vampire bat, the lower the return rate for retaining more blood by the same bat (Wilkinson, [1984]). In yeast, about $99 \%$ of the digested sugars generated from invertase production dissipates away from the producer cells, which suggests that the benefits conferred by invertase saturates once the amount of invertase in the group surpasses a certain level (Gore et al., [2009]).

The puzzle posed by redundancy is largely due to the effect of diminishing returns on group tasks. Specifically, redundancy evolves when the reward for being a cooperator seems negligible once the number of cooperators exceeds a

alleles are expected to be unstable because they are vulnerable to the evolution of defectors that possess the identification tag but fail to produce the costly toxin (Birch, [2017]). 
threshold value, say, $k$. Due to diminishing returns, having $k+1$ as opposed to $k$ cooperators does not considerably increase the chance that the group task will be completed successfully. The overall puzzle is that organisms in nature, such as bees and microbes, cooperate even when they belong to groups that have more than $k$ cooperators. According to Birch's ([2012]) hypothesis, even though $k$ cooperators may be sufficient to complete a group task, there may be more than $k$ cooperators because within-group policing increases the cost of being a non-cooperator. Policing thus makes cooperation relatively cheaper which, in turn, enables the evolution of redundancy.

The puzzle posed by redundancy becomes more challenging if the focal individual is 'well-informed' in the sense that the individual possesses complete information about the amount of cooperators in the group. For the sake of simplicity, suppose that a group task, such as hunting a mammoth, requires exactly $k$ cooperators for the task to be performed successfully. In the absence of policing, a well-informed individual is only expected to cooperate when the group contains $k-1$ cooperators. If there are $k$ or more cooperators, becoming a cooperator is unwarranted because the group already has enough cooperators to hunt the mammoth successfully. Moreover, hunting is a risky endeavor, which makes cooperation costly. Alternatively, if the number of cooperators is strictly lower than $k-1$, becoming a cooperator would be pointless because the hunt would fail either way. ${ }^{7}$ Accordingly, in the absence of policing, being well-informed about the strategies of the other players undermines the evolution of cooperation.

In contrast, redundancy might evolve without the need of coercive behaviors if the group members are ignorant about the strategies of the other members.

\footnotetext{
${ }^{7}$ The assumption that hunting a mammoth requires exactly $k$ cooperators might strike the reader as being too unrealistic. However, as argued in Section 3.2, formal results involving public goods games suggest that relaxing this assumption does not alter the qualitative behavior of the game.
} 
When individuals do not know what others will do, it is as if the individuals have to decide whether they will cooperate while being behind some form of 'Darwinian veil of ignorance' (Skyrms, [1996]). Even without knowing the strategy of the other group members, there are incentives for both cooperating and free-riding. On the one hand, individuals have an incentive to cooperate because completing the group task is beneficial for them. That is, having $k$ or more cooperators produces a benefit that is shared by the group, such as the spoils of the hunt. On the other hand, cooperation is costly and, for this reason, it would be preferable for an individual to free-ride on others. In other words, an individual would be better off if it could obtain the benefits from the group task (e.g., food) without having to pay the costs. Accordingly, without knowing what others will do, individuals are expected to bet on cooperating with a certain probability.

There is a parallel between the discussion above and the evolution of flexible behaviors (Sterelny, [2003]). At first glance, organisms living in heterogeneous environments are expected to exhibit some level of behavioral flexibility because organisms that adopt invariant strategies would be penalized in such environments. However, as Sterelny ([2003]) observes, environmental heterogeneity selects for variable response 'only if that heterogeneity is cued to the organism' (p. 12). As a result, organisms living in an 'informationally opaque' environment - in the sense that the environment is intractably complex for them-'must settle for a single best-overall response' (p. 12). Accordingly, flexible behaviors are more likely to evolve when organisms live in 'informationally transparent' environments. A similar point can be made about group tasks. If the player's strategies are 'informationally opaque' during group tasks, players are expected to cooperate with an intermediary probability. In contrast, if the strategies of other players are 'informationally transparent' for an individual, 
this individual is only expected to voluntarily cooperate when its contribution is indispensable for completing the group task. ${ }^{8}$

In the next section, a particular type of public goods game is used to illustrate the social dilemma posed by group tasks when players' strategies are informationally opaque. This game can be understood as formalizing the argument presented above. The next section thus provides further support for the claim that individuals are expected to cooperate with a certain probability when they lack information about the strategies of the other players.

\subsection{Modeling group tasks with a public goods game}

Suppose individuals form groups with $N$ members. Cooperators pay a cost of $c$ in order to produce the public good. Every member of the group obtains a benefit $b(>c)$ if and only if the number of producers in the group is equal or higher than the threshold value $\tau$, where $1<\tau<N$. Accordingly, receiving the benefit $b$ means that the group successfully completed the group task. Players cooperate with a probability $x \in[0,1]$ and free-ride with a probability $1-x$. This is a 'simultaneous' game in the sense that players choose their strategies without knowing the strategies of the other players. ${ }^{9}$

In order to determine whether this game admits an Evolutionary Stable Strategy (ESS), suppose the focal player cooperates with a chance $y$ and the other $N-1$ players cooperate with a chance $x$. The expected fitness of the focal player is:

$$
W(y ; x)=\sum_{j=\tau}^{N-1} p_{j}(x) b+y \cdot g(x),
$$

\footnotetext{
${ }^{8}$ I thank one of the referees for suggesting this parallel with Sterelny's ([2003]) work.

${ }^{9}$ This game relies on Bach et al.'s ([2006]) framework for 'threshold games'. In addition to Bach et al. ([2006]), several papers have studied similar games, including Archetti ([2009]); Pacheco et al. ([2009]) and Archetti and Scheuring ([2011]). See Archetti and Scheuring ([2012]) for a review of the literature on threshold games.
} 
where $p_{j}(x)$ is the probability that $j$ of the other $N-1$ players will cooperate:

$$
p_{j}(x)=\left(\begin{array}{c}
N-1 \\
j
\end{array}\right) x^{j}(1-x)^{N-1-j},
$$

and $g(x)$ is the expected gain when the focal player cooperates:

$$
g(x)=p_{\tau-1}(x) b-c .
$$

The strategy $x$ is a Nash Equilibrium (NE) when $W(x ; x) \geq W(y ; x)$, for all $y \neq x$. Accordingly, the pure strategy of always cooperating $(x=1)$ is not a NE, but always defecting $(x=0)$ is. Furthermore, $x$ is a mixed NE if $g(x)=0$; that is, when $p_{\tau-1}(x)=c / b$. A strategy $x$ is an Evolutionary Stable Strategy (ESS) if a population playing $x$ can resist being invaded by a few mutants playing any other strategy (Broom et al., [1997]). As Bach et al. ([2006]) prove, a mixed strategy $x$ in this type of game is an ESS when $g(x)=0(x$ is a NE) and $g^{\prime}(x)<0$.

The behavior of the function $p_{\tau-1}(x)$ dictates whether the game admits a mixed ESS. Since $p_{\tau-1}(x)$ is the mass function of a binomial distribution (Eq. 2), this function possesses a single maximum when $x_{*}=(\tau-1) /(N-1)$. Accordingly, if $p_{\tau-1}\left(x_{*}\right)<c / b$, the game does not possess a mixed NE. If $p_{\tau-1}\left(x_{*}\right)>c / b$, the game admits two mixed NE, $x_{-}<x_{*}$ and $x_{+}>x_{*}$. Of these two mixed NE, $x_{+}$is the only ESS because $g^{\prime}\left(x_{+}\right)<0$. As an example, consider a group of 10 individuals in which only 4 cooperators are required to generate the group benefit, and that $b=5 c$. The function $p_{\tau-1}(x)$ is plotted below: 


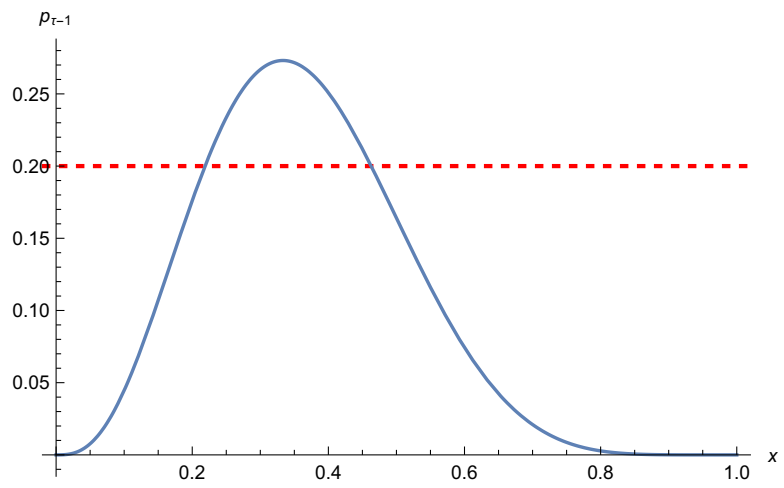

The dashed line intercepts the two mixed NE: $x_{-} \approx 0.22$ (unstable) and $x_{+} \approx$ 0.46 (ESS). Accordingly, group members are expected to cooperate about $46 \%$ of the time at ESS. If a few mutants evolve that cooperate, say, $30 \%$ or $60 \%$ of the time, these mutants will be selected against. ${ }^{10}$ This game thus nicely illustrates how group tasks generate incentives for both cooperating and freeriding. As a result of these incentives, if the benefit generated by the group task is sufficiently high, individuals are expected to cooperate with a probability between zero and one, despite their lack of knowledge about the strategies of others.

One potential limitation of the public goods game introduced in this section is that the group benefit is modeled by a 'sharp' function: the group benefit is only produced when there are $\tau$ or more cooperators (Figure 1A). ${ }^{11}$ In contrast, the benefit generated by group tasks likely follows a 'smooth' function before reaching the threshold $\tau$ in the sense that the group benefit gradually increases as the number of cooperators raises from zero to $\tau$ (Figure 1B). For example, it seems that the thicker the biofilm the lower the chance that an antimicrobial agent will successfully penetrate the biofilm (Stewart and Costerton, [2001]). However, as Bach et al. ([2006]) prove, using a smoother function does not

\footnotetext{
${ }^{10}$ Accordingly, finding ESSs also indicates how the game evolves over time. In particular, ESSs are attractors of replicator dynamics (Hofbauer and Sigmund, [1998]).

${ }^{11}$ I thank one of the referees for pointing this out.
} 


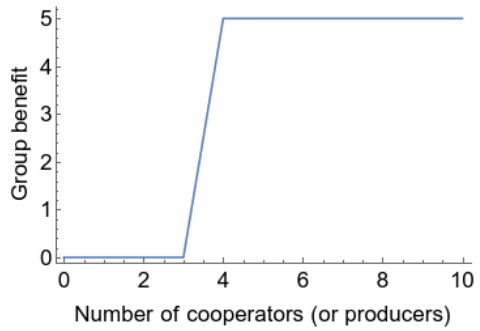

(A)

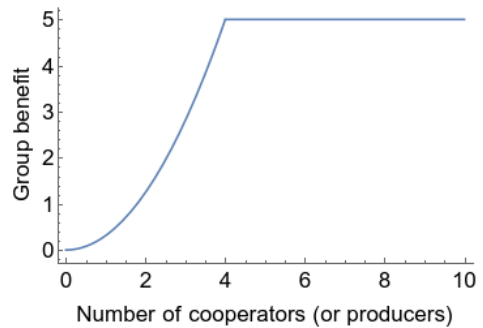

(B)

Figure 1: (A) Sharp condition. The resulting group benefit function is sharp in the sense that it drastically changes its value when the number of cooperators increases from zero to the threshold value $\tau=4$. (B) Smooth condition. Group benefit increases smoothly as the number of cooperators reaches the threshold $\tau=4$.

change the qualitative behavior of threshold games. More precisely, the conditions for a mixed ESS mentioned above continue to hold even when the group benefit varies with the number of cooperators as illustrated by Figure $1 \mathrm{~B} .{ }^{12} \mathrm{In}$ this way, despite its simplicity, modeling the group benefit as a sharp function provides a reasonable approximation of the type of social dilemma associated with group tasks when individuals lack information about the strategy of other players.

\subsection{Group tasks under harsh environments}

The previous two sections made the case that individuals possess incentives to both cooperate and defect in group tasks when individuals do not know what others will do (Sections 3.1 and 3.2). As a result, it is expected that individuals will cooperate with a certain probability. But how high should this probability be?

A reasonable hypothesis is that, even without knowing if others will coop-

\footnotetext{
${ }^{12}$ Similarly, Archetti and Scheuring ([2011]) show that the replicator dynamics of a threshold game, the Volunteer's Dilemma, admits a mixed equilibrium regardless of whether the group benefit is modeled by an S-shaped function (smooth condition) or a Heaviside step function (sharp condition).
} 
erate, the harsher the environment the higher the incentive to bet on cooperating. Organisms live in 'harsh' environments when the cost of not completing a particular group task is sufficiently high (Dugatkin, [1997]). For the sake of illustration, suppose a group of hunters lives in an environment in which mammoths are the only type of animal available to hunt, and that a critical number of volunteers is required to hunt a mammoth successfully. This environment is extremely harsh because, if there are not enough volunteers, everyone pays a high price; that is, the whole group fails to obtain enough food to survive. ${ }^{13}$ The effect of harsh environments on the level of cooperation within groups is well-documented in several species (Bourke, [2011]). For example, a comparative study involving distinct species of starlings shows that cooperative breeding is positively correlated with semi-arid savanna habitats and environments with temporally variable rainfall (Rubenstein and Lovette, [2007]). Moreover, harsh environments are crucial for explaining the social behaviors of microbes.

Microbes are notorious for their tenacity in hostile environments. In the case of bacteria, this is largely attributed to their formation of 'biofilms', which allow them to withstand a wide range of environmental perturbations, including antimicrobial treatments (Stewart and Costerton, [2001]), host immune defenses (Fux et al., [2005]), UV radiation (Elasri and Miller, [1999]), and protozoan predators (Matz and Kjelleberg, [2005]). Moreover, the resilience of biofilms and other microbial groups often require a critical number of cooperators to manifest (Rauch et al., [2017]). For instance, a certain number of bacterial cells need to stick together in order to form a microcolony that is large enough to

\footnotetext{
${ }^{13}$ Bourke ([2011]) advances a related idea. He suggests that selfish members within a group might limit their own reproduction if they 'damage group growth, survival, or productivity to such an extent that they impair their own reproductive success' (p. 140). Transmissible cancers, such as the canine transmissible venereal tumor, illustrate such a possibility. The reason is cancerous cells (selfish members) can drive their hosts - and, consequently, themselves - to extinction if cancerous cells do not limit their own reproductive success. In this way, transmissible cancers can be thought of as living in extremely harsh environments. I thank one of the referees for suggesting this comparison.
} 
deter protozoan grazers (Matz and Kjelleberg, [2005]). Pseudomonas aeruginosa biofilms collapse if enough cells do not contribute to the construction of the biofilm (Rainey and Rainey, [2003]). In yeast, a higher proportion of cooperators can increase the resilience of the population against perturbations (Sanchez and Gore, [2013]). Accordingly, harsh environments can foster cooperation in microbial groups by increasing the cost of not having enough cooperators to maintain the group.

The concept of harsh environments helps distinguish two types of costs associated with group tasks. First, there is the 'individual'-level cost for cooperating. For instance, microbial cells often cooperate by producing public goods, such as polymers, which incur a metabolic cost to the producer. Moreover, there is a 'group'-level cost when there are not enough cooperators to complete a group task, such as hunting a mammoth. Groups are considered to be living in 'harsh environments' when the group-level costs are sufficiently high relative to the individual-level costs. In contrast, groups are considered to be living in 'mild environments' when the group-level cost is low relative to the individual-level cost (Dugatkin, [1997]). When the strategies of the other players are unknown, mild environments increase the incentive for players to bet on free-riding; in contrast, harsh environments increase the incentive to bet on cooperating.

When groups live in harsh environments, redundancy might evolve as a byproduct from selection at the organism level. Without knowing what others will do, players must rely on the type of environment to decide how much they will bet on cooperating. The harsher the environment, the higher their incentive to bet on cooperating. However, by increasing the chance that individuals will cooperate, harsh environments indirectly increase the chance of the evolution of redundant groups. In other words, redundant groups initially evolve not because there is selection 'for' redundancy. Instead, harsh environments select for 
individuals that are willing to pay for the individual-level cost for cooperating despite the possibility of being taken advantage of by free-riders. As an incidental consequence, harsh environments increase the chance that groups will contain more cooperators than strictly necessary to complete the group task.

As an example, consider the threshold game discussed previously (Section 3.2). By manipulating the costs and benefits in this game, we can simulate how the mixed ESS changes when individuals live in harsher environments. Since individuals at the mixed ESS cooperate around $46 \%$ of the time, the group might end up with different numbers of cooperators. Specifically, the chance that $i$ out of $N$ members of the group are cooperators at ESS is summarized by the graph below:

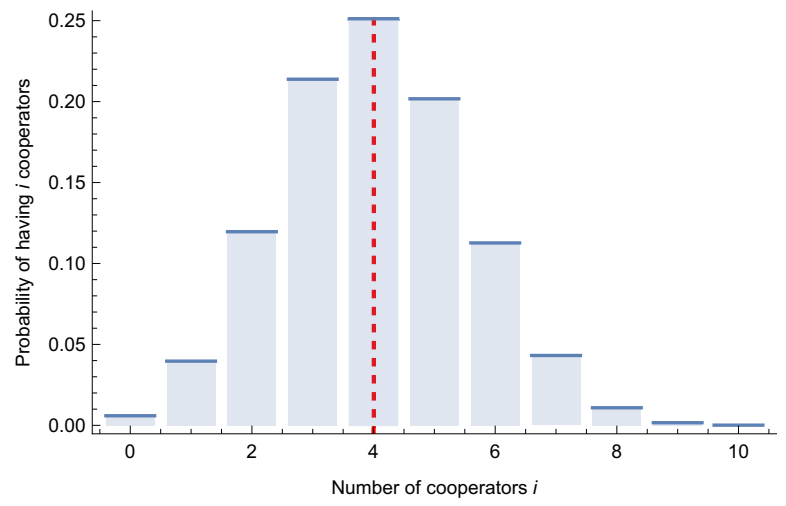

The level of 'harshness' of an environment is due to the difference in the expected payoff between when the group task is successfully performed from when it fails to be completed. Accordingly, in order to simulate a harsher environment, suppose $b=100 c$ (instead of $b=5 c$ ). The mixed ESS is now $\approx 0.74$ (instead of $\approx 0.48)$. Moreover, the probability that the group will have at least four cooperators to complete the group task successfully is, as expected, considerably higher: 


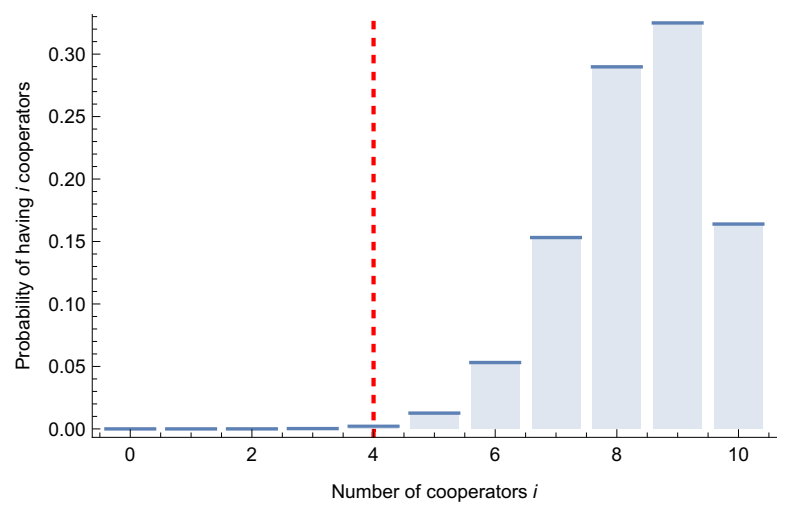

As the environment becomes harsher, the probability distribution is supposed to shift to the right because individuals have a higher incentive to complete the group task. As an incidental consequence, the probability that a redundant group (that is, a group with more than four cooperators) will evolve also increases. For instance, in the last graph, the chance of having eight cooperators is higher than the chance of having four cooperators in the group even though only four cooperators are required to complete the group task successfully. Additionally, redundancy evolves despite the fact that the players do not know the strategy of the other group members.

Harsh environments can only account for redundancy if group members possess limited information about the strategy of others. The incentive to cooperate increases in harsh environments because the group faces the uncertainty of not having enough cooperators to produce a vital collective benefit, such as protection against predators. In contrast, an individual that knows the strategy of others will only cooperate when the number of cooperators is just below the threshold value required to complete the group task. As a result, in the absence of within-group policing, being well-informed about the strategies of other players would undermine the evolution of redundancy.

Policing can be thought of as emulating harsh environments within groups. According to Birch ([2012]), policing can enable the evolution of redundancy by 
increasing the costs associated with free-riding, which makes cooperation more cost-effective. Similarly, harsh environments coupled with lack of information perform a similar role: the risk of failing to produce a critical group benefit adds a further cost to being a free-rider. ${ }^{14}$ In this way, the explanation proposed in this paper can be viewed as complementing Birch's account. In systems in which players have limited information about the strategies of others, redundancy might be due to harsh environments. ${ }^{15}$ Nevertheless, when groups contain wellinformed individuals, harsh environments can no longer explain the evolution of redundancy. In such cases, as suggested by Birch ([2012]), the evolution of redundancy might require the presence of coercive behaviors within the group.

\section{Conclusion}

Redundancy is probably adaptive at the group-level because redundant groups tend to be more resilient to environmental perturbations (e.g., predation) than non-redundant groups (Charbonneau et al., [2017]). Still, the group-level benefits due to redundancy fail to explain why within-group selection does not favor free-riders (Birch, [2012]). The main take-home message of this paper is that harsh environments, when coupled with lack of information about the strategy of the other players, increase the chance that the reproductive interests of the group and its members align with each other. At the group-level, redundancy is adaptive because it increases the resilience of groups to hostile environments; at

\footnotetext{
${ }^{14}$ The suggestion that policing can be viewed as a type of harsh environment produced by group members appears in Dugtakin's ([2000]) discussion of mutualism in humans. In order to motivate his point, Dugatkin cites Ridley's ([1997]) idea that the invention of the dart thrower not only enabled humans to kill large mammals more easily, but it also enabled humans to threaten others that would not cooperate in the hunt. As Dugatkin puts it, "[t]he newest element of the harsh environment that might have boomeranged against a cheater was the fellow next to him, who not only wasn't happy that the cheater skipped out on the hunt while still expecting a meal, but who also happened to be carrying a rather dangerous weapon' (Dugatkin, [2000], p. 99).

${ }^{15}$ Despite our cognitive abilities, humans often see themselves in situations in which a decision has to be made without knowing what others will do. The bystander effect nicely illustrates this type of situation (Darley and Latané, [1968]; Diekmann, [1985]).
} 
the individual-level, redundancy evolves because individuals are shortsighted.

\title{
Acknowledgments
}

I gratefully acknowledge the constructive suggestions of the anonymous referees. I also thank the DC/Maryland History and Philosophy of Biology discussion group (especially Eric Saidel), and the audience from the PSA 2018 meeting for providing valuable feedback on earlier versions of this paper.

\author{
Makmiller Pedroso \\ Philosophy 8 Religious Studies \\ Towson University \\ Towson, USA \\ mpedroso@towson.edu
}

\section{References}

Albert, R., Jeong, H., and Barabási, A. [2000]. Error and attack tolerance of complex networks. Nature, 406, pp. 378-382.

Anderson, C. and Franks, N. [2001]. Teams in animal societies. Behavioral Ecology, 12, pp. 534-540.

Anderson, C., Franks, N., and McShea, D. [2001]. The complexity and hierarchical structure of tasks in insect societies. Animal Behaviour, 62, pp. 643-651.

Anderson, C., Theraulaz, G., and Deneubourg, J.-L. [2002]. Self-assemblages in insect societies. Insectes Sociaux, 49, pp. 99-110.

Archetti, M. [2009]. Cooperation as a volunteer's dilemma and the strategy of conflict in public goods games. Journal of Evolutionary Biology, 22, pp. 21922200. 
Archetti, M. and Scheuring, I. [2011]. Coexistence of cooperation and defection in public goods games. Evolution, 65, pp. 1140-1148.

Archetti, M. and Scheuring, I. [2012]. Game theory of public goods in one-shot social dilemmas without assortment. Journal of Theoretical Biology, 299, pp. 9-20.

Bach, L., Helvik, T., and Christiansen, F. [2006]. The evolution of n-player cooperation - threshold games and ess bifurcations. Journal of Theoretical Biology, 238, pp. 426-434.

Birch, J. [2012]. Collective action in the fraternal transitions. Biology and Philosophy, 27, pp. 363-380.

Birch, J. [2017]. The Philosophy of Social Evolution. Oxford University Press.

Bourke, A. [2011]. Principles of Social Evolution. Oxford University Press.

Bowles, S. and Gintis, H. [2011]. A Cooperative Species: Human Reciprocity and its Evolution. Princeton University Press.

Brennan, J. [2016]. The ethics and rationality of voting. In Zalta, E. N., editor, The Stanford Encyclopedia of Philosophy. Metaphysics Research Lab, Stanford University, winter 2016 edition.

Broom, M., Cannings, C., and Vickers, G. [1997]. Multi-player matrix games. Bulletin of Mathematical Biology, 59, pp. 931-952.

Calcott, B. [2008]. The other cooperation problem: Generating benefit. Biology E Philosophy, 23, pp. 179-203.

Charbonneau, D. and Dornhaus, A. [2015]. When doing nothing is something: How task allocation strategies compromise between flexibility, efficiency, and inactive agents. Journal of Bioeconomics, 17, pp. 217-242. 
Charbonneau, D., Sasaki, T., and Dornhaus, A. [2017]. Who needs 'lazy' workers? Inactive workers act as a 'reserve' labor force replacing active workers, but inactive workers are not replaced when they are removed. PloS One, 12, pp. 1-20.

Darley, J. and Latané, B. [1968]. Bystander intervention in emergencies: diffusion of responsibility. Journal of Personality and Social Psychology, 8, pp. 377-383.

Dawkins, R. [1976]. The Selfish Gene. Oxford University Press, Oxford.

Diekmann, A. [1985]. Volunteer's dilemma. Journal of Conflict Resolution, 29, pp. 605-610.

Dugatkin, L. [1997]. Cooperation Among Animals: An Evolutionary Perspective. Oxford University Press.

Dugatkin, L. [2000]. Cheating Monkeys and Citizen Bees. Harvard University Press.

Elasri, M. and Miller, R. [1999]. Study of the response of a biofilm bacterial community to UV radiation. Applied and Environmental Microbiology, 65, pp. 2025-2031.

Flemming, H., Wingender, J., Szewzyk, U., Steinberg, P., Rice, S., an] Kjelleberg, S. (2016). Biofilms: an emergent form of bacterial life. Nature Reviews Microbiology, 14, pp. 563-575.

Fux, C., Costerton, J., Stewart, P., and Stoodley, P. [2005]. Survival strategies of infectious biofilms. Trends in Microbiology, 13, pp. 34-40.

Gore, J., Youk, H., and Van Oudenaarden, A. [2009]. Snowdrift game dynamics and facultative cheating in yeast. Nature, 459, pp. 253-256. 
Hofbauer, J. and Sigmund, K. [1998]. Evolutionary Games and Population Dynamics. Cambridge University Press.

Hölldobler, B. and Wilson, E. [2009]. The Superorganism: The Beauty, Elegance, And Strangeness of Insect Societies. WW Norton \& Company.

Krause, J. and Ruxton, G. [2002]. Living in Groups. Oxford University Press.

Matz, C. and Kjelleberg, S. [2005]. Off the hook-how bacteria survive protozoan grazing. Trends in Microbiology, 13, pp. 302-307.

Nowak, M., Boerlijst, M., Cooke, J., and Maynard Smith, J. [1997]. Evolution of genetic redundancy. Nature, 388, pp. 167-171.

Okasha, S. [2006]. Evolution and the Levels of Selection. Oxford University Press, Oxford.

Pacheco, J., Santos, F., Souza, M. O., and Skyrms, B. [2009]. Evolutionary dynamics of collective action in n-person stag hunt dilemmas. Proceedings of the Royal Society of London B: Biological Sciences, 276, pp. 315-321.

Pedroso, M. [2018]. The impact of population bottlenecks on the social lives of microbes. Biological Theory, 13, pp. 190-198.

Queller, D. and Strassmann, J. [2009]. Beyond society: the evolution of organismality. Philosophical Transactions of the Royal Society B, 364, pp. 31433155.

Rainey, P. and Rainey, R. [2003]. Evolution of cooperation and conflict in experimental bacterial populations. Nature, 425, pp. 72-74.

Ratnieks, F. and Wenseleers, T. [2008]. Altruism in insect societies and beyond: voluntary or enforced? Trends in Ecology 83 Evolution, 23, pp. 45-52. 
Rauch, J., Kondev, J., and Sanchez, A. [2017]. Cooperators trade off ecological resilience and evolutionary stability in public goods games. Journal of The Royal Society Interface, 14, p. 20160967.

Ridley, M. [1997]. The Origins of Virtue. Viking.

Rubenstein, D. and Lovette, I. [2007]. Temporal environmental variability drives the evolution of cooperative breeding in birds. Current Biology, 17, pp. 14141419.

Sanchez, A. and Gore, J. [2013]. Feedback between population and evolutionary dynamics determines the fate of social microbial populations. PLOS Biology, 11, pp. $1-9$.

Skyrms, B. [1996]. Evolution of the Social Contract. Cambridge University Press.

Sterelny, K. [2003]. Thought in a Hostile World: The Evolution of Human Cognition. Wiley-Blackwell.

Stewart, P. and Costerton, J. [2001]. Antibiotic resistance of bacteria in biofilms. The Lancet, 358, pp. 135-138.

Travisano, M. and Velicer, G. [2004]. Strategies of microbial cheater control. Trends in Microbiology, 12, pp. 72-78.

Vlamakis, H., Aguilar, C., Losick, R., and Kolter, R. [2008]. Control of cell fate by the formation of an architecturally complex bacterial community. Genes E) Development, 22, pp. 945-953.

Westall, F., de Wit, M., Dann, J., van der Gaast, S., de Ronde, C., and Gerneke, D. [2001]. Early archean fossil bacteria and biofilms in hydrothermallyinfluenced sediments from the barberton greenstone belt, south africa. Precambrian Research, 106, pp. 93-116. 
Wilkinson, G. [1984]. Reciprocal food sharing in the vampire bat. Nature, 308,

pp. 181-184. 